\title{
BREVE ANÁLISIS DE LA PRESENCIA DE LA PERSPECTIVA DE GÉNERO EN LOS INSTRUMENTOS INTERNACIONALES DE DERECHOS HUMANOS Y EN LAS SENTENCIAS DE LA CORTE INTERAMERICANA
}

\author{
Mónica Anís - Graciela Gómez Vara - Verónica Torres ${ }^{1}$
}

En este breve trabajo nos proponemos analizar las implicancias de algunos instrumentos internacionales de derechos humanos en lo relativo al derecho a la igualdad de mujeres y hombres y el afianzamiento de la perspectiva de género en el sistema interamericano.

En este sentido, en primer lugar, recurriremos al concepto de género, ya que será de utilidad para el desarrollo y análisis de este trabajo. Para ello partimos de la base de que el concepto alude tanto al conjunto de características como a los roles, funciones y estereotipos impuestos de manera dicotómica a cada sexo a través de los procesos de socialización, los cuales han sido perpetuados por estructuras e instituciones patriarcales y cuya repercusión general es que todo lo masculino se convierte en el modelo de lo humano. ${ }^{2}$ El concepto de género, y su perspectiva, se han incorporado al sistema internacional de los derechos huma-

${ }^{1}$ El presente trabajo se realiza en el marco del PI SGCyT-UNNE 11g002, del cual las autoras forman parte. en la Facultad de Derecho y Ciencias Sociales y Políticas de la UNNE, Mónica Anís es profesora del Seminario de Derechos Humanos y Secretaria del H. Consejo Directivo, Graciela Gómez Vara es profesora del Seminario de Orientación en Derecho Civil y de Derecho Procesal Civil y Comercial, Verónica Torres es profesora de Derecho Comercial I curso y Decana.

${ }^{2}$ Según Marta Lamas (2002:36-39) el papel o rol de género se configura con el conjunto de normas y prescripciones que dictan la sociedad y la cultura sobre el comportamiento femenino o masculino. Si bien esta autora sostiene que hay diferencias culturales, de clases sociales o grupo étnico de las personas, se puede sostener una división básica que corresponde a la división sexual del trabajo más primitiva: las mujeres son las encargadas de los hijos y el hogar por lo que el ámbito que les compete es el privado identificado obviamente con el hogar y la familia, mientras que se identifica lo masculino con el ámbito público. Esta dicotomía estuvo prevista por ejemplo en las normas del código civil argentino redactado por Vélez Sarsfield, en que se evidenciaba claramente estas diferencias en las que la dicotomía en los roles masculino femenino establece estereotipos que condicionan los papeles y limitan muchas veces las potencialidades 
nos, permitiendo grandes ventajas y posibilidades para la efectiva tutela de todas las personas, y concretamente de las mujeres, y ha permitido desnudar que la tan mentada dicotomía sexual es impuesta socialmente a través de roles y estereotipos que hacen aparecer a los sexos como diametralmente opuestos.

Es así que, a partir de una exagerada importancia que se da a las diferencias biológicas reales, se construyen roles para cada sexo y las características con que se define a uno u otro gozan de distinta jerarquía, en el sentido de que legitimen la subordinación del sexo femenino que no es dada por la naturaleza. Es decir, mientras que el concepto «sexo» podría afirmarse que es biológico, el de género es una construcción social. Esta distinción es muy importante, ya que nos permite entender que no hay nada natural en los roles y características sexuales y que por lo tanto pueden ser transformados.

La perspectiva de género permite advertir que la identidad femenina es construida socialmente, que resulta de los roles y atribuciones que cada sociedad en cada época otorga a cada sexo y también que es una estructura que divide el trabajo en aquel que se realiza en el hogar y aquel que se realiza en la esfera pública; legitima la desigualdad de autoridad y poder entre hombres y mujeres dentro de cada clase social, etnicidad, raza, edad o grupo humano y jerarquiza los valores atribuidos a los hombres, institucionalizándolos como paradigma de la humanidad.

El ámbito jurídico no escapa a esta realidad, ya que el derecho desempeña y ha desempeñado un importante y elemental papel en la desigualdad de las mujeres: como no es solo normatividad, está compuesto por prácticas sociales y por discursos legitimantes emanados, en su mayoría, de las estructuras de poder en una sociedad (Alicia Ruiz, 2009:115-121). Así, el origen de muchas desigualdades que se hallan en la estructura social actual aparece en muchas leyes que no tienen en cuenta descripciones de la realidad género, que incluyan a las mujeres y a las relaciones de poder articuladas entre ellas y los hombres. Por ello, se hace necesario incorporar la perspectiva de género en todas las interpretaciones y análisis de la realidad, la legislación y el ejercicio de derechos, en el diseño de estrategias y en la evaluación de acciones tendientes a lograr la igualdad real.

Ahora bien, nuestro país no escapa al impulso mundial de los Estados de derecho creados al amparo de las revoluciones burguesas donde los conjuntos 
normativos, las prácticas y los discursos jurídicos plasmaban en concreto las situaciones de desigualdad entre hombres y mujeres hasta bien entrado el siglo XX.

Luego de las guerras mundiales, y con las primeras declaraciones universales o continentales, la tendencia normativa pretendió modificar esta situación en el plano meramente legislativo puesto que las prácticas, los estereotipos culturales y las políticas públicas no tendían puentes para la concreción real de la igualdad de género.

Es necesario aclarar que, sin necesidad de analizar instrumentos específicos que tratan las situaciones de las mujeres, todos los instrumentos incluidos en la redacción de artículo 75 inciso 22 de nuestra Constitución Nacional poseen cláusulas relativas a evitar la discriminación por sexo.

Así, el artículo 2 inc. 1 de la Declaración Universal de Derechos Humanos dice: "Toda persona tiene todos los derechos y libertades proclamados en esta Declaración, sin distinción alguna de raza, color, sexo, idioma, religión, opinión política o de cualquier otra índole, origen nacional o social, posición económica, nacimiento o cualquier otra condición”. Además consagra en el artículo 7 que: “Todos son iguales ante la ley y tienen, sin distinción, derecho a igual protección de la ley. Todos tienen derecho a igual protección contra toda discriminación que infrinja esta Declaración y contra toda provocación a tal discriminación".

Por su parte, la Convención Americana de Derechos Humanos consagra en el artículo 2 que:

Los Estados Partes en esta Convención se comprometen a respetar los derechos y libertades reconocidos en ella y a garantizar su libre y pleno ejercicio a toda persona que esté sujeta a su jurisdicción, sin discriminación alguna por motivos de raza, color, sexo, idioma, religión, opiniones políticas o de cualquier otra índole, origen nacional o social, posición económica, nacimiento o cualquier otra condición social.

Además, en el artículo 17 inc. 2 se reconoce el derecho del hombre y la mujer a contraer matrimonio y a fundar una familia si tienen la edad y las condiciones requeridas para ello por las leyes internas, en la medida en que estas no afecten al principio de no discriminación establecido en esta Conven- 
ción. Y en el inciso 4 que los Estados partes deben tomar medidas apropiadas para asegurar la igualdad de derechos y la adecuada equivalencia de responsabilidades de los cónyuges en cuanto al matrimonio, durante el matrimonio y en caso de disolución del mismo. En caso de disolución, se adoptarán disposiciones que aseguren la protección necesaria de los hijos, sobre la base única del interés y conveniencia de ellos.

De igual manera, la convención americana consagra en su artículo 2 que:

Cada uno de los Estados Partes en el presente Pacto se compromete a respetar y a garantizar a todos los individuos que se encuentren en su territorio y estén sujetos a su jurisdicción los derechos reconocidos en el presente Pacto, sin distinción alguna de raza, color, sexo, idioma, religión, opinión política o de otra índole, origen nacional o social, posición económica, nacimiento o cualquier otra condición social. Y en su artículo 3 compromete a los Estados partes a garantizar a hombres y mujeres la igualdad en el goce de todos los derechos civiles y políticos enunciados en el Pacto.

Finalmente, la Convención sobre la eliminación de todas las formas de discriminación contra la Mujer (en adelante CEDAW), ${ }^{3}$ supone la Carta Magna femenina en cuanto al reconocimiento de los derechos de las mujeres $\mathrm{y}$, en su consecuencia, de la aplicación del principio de igualdad y prohibición de discriminación por razón de género. Su adopción en el seno de Naciones Unidas constituye un avance superlativo en el proceso del reconocimiento de los derechos humanos de las mujeres introduciendo la perspectiva de género en un instrumento internacional de derechos humanos.

La CEDAW es la culminación de más de treinta años de trabajo realizado por expertos de las Naciones Unidas, y que, como recuerda Caputti (2011;III:84), también aparece como aspiración fundacional de la ONU desde el preámbulo de la Carta de San Francisco, donde se fija como objetivos básicos la reafirmación de la " $f e$ en los derechos fundamentales del hombre, en la dignidad y el valor de la persona humana, en la igualdad de derechos de mujeres y hombres". Además el artículo $1^{\circ}$ de dicha Carta se establece como uno de los

${ }^{3}$ Es la abreviatura habitualmente utilizada para esta convención por sus siglas en inglés. 
propósitos de la organización de las naciones unidas el desarrollo y respeto de los derechos de todas las personas, vedando toda distinción por motivos de raza, sexo, idioma o religión.

La CEDAW reúne en un instrumento internacional de derechos humanos, muchas de las disposiciones de instrumentos anteriores de la ONU relativas a la discriminación contra la mujer. Se dice que es la Carta Internacional de los Derechos Humanos de las Mujeres porque es el primer instrumento internacional que incluye muchos derechos humanos específicos de las mujeres al prohibir todas las formas de discriminación por razones de sexo.

Si bien es cierto que muchos de los instrumentos internacionales comentados en este trabajo prohíben la discriminación por razones de sexo y garantizan la igualdad de todos los seres humanos, en la práctica esto no ha sido suficiente para garantizar a las mujeres la protección de sus derechos.

Por su parte, Facio ${ }^{4}$ entiende que esto se produce porque si bien muchos instrumentos establecen prohibiciones respecto de las discriminaciones en razón de sexo, no es menos cierto que la perspectiva de esos instrumentos no deja de ser androcéntrica, ya que está pensada a partir de la realidad y de las necesidades masculinas.

Por esta circunstancia, la CEDAW ha producido un cambio de paradigma en lo relativo al derecho a la igualdad ya que asume una realidad: la histórica desigualdad entre hombres y mujeres.

Es así que la CEDAW es el primer instrumento internacional de derechos humanos que toma como punto de partida esa histórica desigualdad y por ende, aunque todavía no se hablaba de género o perspectiva de género en el momento en que fue discutida, sí se puede decir que es un instrumento con perspectiva de género.

Esta convención se aprobó el 18 de diciembre de 1979 por la Asamblea General de las Naciones Unidas y entró en vigor como tratado internacional el 3 de setiembre de 1981, tras su ratificación por 20 países. Es importante destacar que después de la Convención de los Derechos del Niño, esta es la convención que logró mayor cantidad de ratificaciones por parte de los estados y también es célebre por la cantidad de reservas que esos mismos países han opuesto a sus postulados.

${ }^{4}$ Texto disponible en: http://www.redtrasex.org.ar/documentos/docsnuevos/ facio_carta_magna_notas_amg.pf 
En esta línea es interesante advertir que algunas de las reservas realizadas por los estados apuntan a cuestiones medulares de la convención tales como la igualdad entre los géneros y algunas reservas dejan a salvo la posibilidad del estado de efectuar discriminaciones en determinados ámbitos o esferas, generalmente en temas concernientes a las relaciones de familia. ${ }^{5}$

El primer artículo de la CEDAW ${ }^{6}$ define lo que se entiende por discriminación contra la mujer, haciendo una distinción interesante respecto de las leyes que según lo establecido pueden ser tachadas de discriminatorias si el resultado de su aplicación es discriminatorio aunque no se haya promulgado con la intención o con el objeto de discriminarla.

En nuestro ordenamiento jurídico, esta definición adquiere singular importancia ya que está incluida en un instrumento de derechos humanos con jerarquía constitucional, por lo cual ningún juez de cualquier instancia en el país puede apartarse de esta definición de discriminación contra la mujer. También es importante destacar que respecto de las posibles definiciones restrictivas que puedan encontrarse en los ordenamientos jurídicos de los estados signatarios de la convención, estos son responsables de adecuarlas al citado artículo $1^{\circ}$ $\mathrm{y}$ al resto del instrumento analizado.

Siguiendo el esquema brindado por Facio podemos sintetizar algunos de los aspectos más importantes de la convención de la siguiente manera:

Establece la obligación concreta de los estados firmantes de adoptar medidas efectivas para eliminar la discriminación contra las mujeres. Según el artículo segundo de la CEDAW, los Estados que ratifiquen esta convención condenan toda forma de discriminación basada en el sexo, y se obligan

${ }^{5}$ Es importante recordar que la Convención de Viena sobre el derecho de los tratados, suscrita el 23 de mayo de 1969 que entró en vigencia el 27 de enero de 1980 prevé mecanismos tendientes a rechazar reservas a los tratados que produzcan como resultados un desnaturalización del tratado, afectando el objeto y fin del mismo. El problema es que este tratado al igual que todas las convenciones internacionales no es retroactivo por lo cual no puede aplicarse a las reservas efectuadas a la Cedaw.

${ }^{6}$ A los efectos de la presente convención, la expresión «discriminación contra la mujer» denotará toda distinción, exclusión o restricción basada en el sexo que tenga por objeto o por resultado menoscabar o anular el reconocimiento, goce o ejercicio por la mujer, independientemente de su estado civil, sobre la base de la igualdad del hombre y la mujer, de los derechos humanos y las libertades fundamentales en las esferas política, económica, social, cultural y civil o en cualquier otra esfera. 
a adoptar medidas concretas y eficaces para hacer efectiva dicha igualdad. Estas medidas se realizarán a través de los cambios legislativos tendientes a modificar las prácticas y costumbres discriminatorias. Ha sido a través de las recomendaciones y comentarios finales del Comité de la CEDAW donde esas obligaciones del Estado se han especificado. Así, por ejemplo, en su Recomendación General $N^{\circ} 21$, el Comité amplía las medidas concretas que deben tomar los estados en relación al matrimonio y las relaciones familiares al establecer, inter alia, que la madre y el padre independientemente de su estado civil deben tener los mismos derechos y responsabilidades respecto a los hijos, ${ }^{7}$ que las leyes o costumbres que no tratan los bienes acumulados durante una relación similar al matrimonio como tratan a la propiedad conyugal, deben derogarse. ${ }^{8}$ Este último caso es importante ya que obliga al Estado no solo a tomar medidas para lograr la igualdad entre hombres y mujeres en cuanto a sus derechos durante el matrimonio y después de su disolución, sino a garantizarles esos mismos derechos a las mujeres que viven en uniones de hecho.

Responsabilidad del Estado y personas privadas en materia de discriminación contra la mujer. La CEDAW, es la primera convención interna-

${ }^{7}$ Artículo 16: “1. Los estados partes adoptarán todas las medidas adecuadas para eliminar la discriminación contra la mujer en todos los asuntos relacionados con el matrimonio y las relaciones familiares y, en particular, asegurarán, en condiciones de igualdad con el hombre: a) el derecho para contraer matrimonio; b) el derecho para elegir libremente cónyuge y contraer matrimonio solo por su libre albedrío y con su pleno consentimiento; c) los derechos y responsabilidades durante el matrimonio y al disolverse éste; d) los derechos y responsabilidades como progenitores, cualquiera que sea su estado civil, en materias relacionadas con sus hijos; en todos los casos, los intereses de los hijos serán la consideración primordial; e) los derechos a decidir libre y responsablemente el número de sus hijos y el intervalo entre los nacimientos y a recibir información, una educación y los medios que les permitan ejercer estos derechos; f) los derechos y responsabilidades respecto de la tutela, curatela, custodia y adopción de hijos, o instituciones análogas cuando quiera que estos conceptos existan en la legislación nacional; en todos los casos, los intereses de los hijos serán la consideración primordial; g) los derechos personales como marido y mujer, entre ellos el derecho a elegir apellido, profesión y ocupación; h) los derechos en el matrimonio en materia de bienes, adquisición, gestión, administración, goce y disposición de los bienes, tanto a título gratuito como oneroso”.

${ }^{8}$ En muchos países, los bienes acumulados durante el amancebamiento no reciben el mismo trato legal que los bienes adquiridos durante el matrimonio. Invariablemente, cuando termina la relación, la mujer recibe una parte considerablemente menor que el hombre. Las leyes y las costumbres sobre la propiedad que discriminan de esta forma a las mujeres casadas o solteras, con o sin hijos, deben revocarse y desalentarse. 
cional que compromete la responsabilidad del estado en la adopción de medidas tendientes a eliminar la discriminación efectuada por personas privadas, empresas o instituciones no estatales u organizaciones. ${ }^{9}$ Este punto es muy importante porque obliga al estado a adoptar políticas integrales tendientes a evitar la discriminación a las mujeres, no solamente en el ámbito estatal sino también en el ámbito privado, ámbito que muchas veces es propicio para que se realicen prácticas discriminatorias. Es oportuno destacar asimismo que la CEDAW fue uno de los primeros instrumentos internacionales en el que se incluyeron las llamadas «medidas de acción afirmativa» ya que el artículo $4^{\circ}$ prevé que la implantación de dichas acciones no se considerará discriminación si tienen por objetivo lograr la igualdad de facto entre hombres y mujeres.

Establece la responsabilidad de los estados signatarios en la eliminación de los patrones socio-culturales que sostienen la discriminación de la mujer. La CEDAW reconoce la importancia de la cultura, tradiciones y prácticas sociales en la construcción de los estereotipos que han repercutido negativamente en todos los ámbitos de la vida de las mujeres y, especialmente el ámbito familiar, en razón de que la discriminación es el resultado de una construcción social, por lo que establece que los Estados deberán adoptar las medidas conducentes a lograr la modificación de los patrones socioculturales con el fin de eliminar los prejuicios y prácticas que se basen en la inferioridad de las mujeres, así como los diferentes roles y funciones asignados a hombres y muje-

${ }^{9}$ Artículo 2: “Los estados partes condenan la discriminación contra la mujer en toda sus formas, convienen en seguir, por todos los medios apropiados y sin dilaciones, una política encaminada a eliminar la discriminación contra la mujer y, con tal objeto, se compromete a: a) consagrar, si aún no lo han hecho, en sus constituciones nacionales y en cualquier otra legislación apropiada el principio de la igualdad del hombre y de la mujer y asegurar por la ley u otros medios apropiados la realización práctica de ese principio; b) adoptar medidas adecuadas, legislativas y de otro carácter, con las sanciones correspondientes, que prohíban toda discriminación contra la mujer; c) establecer la protección jurídica de los derechos de la mujer sobre una base de igualdad con los del hombre y garantizar, por conducto de los tribunales nacionales o competentes y de otras instituciones públicas, la protección efectiva de la mujer contra todo acto de discriminación; d) abstenerse de incurrir en todo acto o práctica de discriminación contra la mujer y velar porque las autoridades e instituciones públicas actúen de conformidad con esta obligación; e) tomar todas las medidas apropiadas para eliminar la discriminación contra la mujer practicada por cualesquiera personas, organizaciones o empresas; f) adoptar todas las medidas adecuadas, incluso de carácter legislativo, para modificar o derogar leyes, reglamentos, usos y prácticas que constituyan discriminación contra la mujer; g) derogar todas las disposiciones penales nacionales que constituyan discriminación contra la mujer”. 
res. En este orden de ideas, la CEDAW alienta la incorporación de las mujeres a la vida política como forma de superar la idea de que el ámbito propio de las mujeres es el ámbito privado, instando a los Estados a eliminar la discriminación en este ámbito y, de este modo, asegurar la participación de las mujeres en la creación de las políticas, leyes de los estados potenciando así la igualdad de oportunidades para el ejercicio de la función pública.

La educación también es un punto clave de la CEDAW, ya esta no se produce en espacios neutrales o aislados. Los estereotipos de género y las relaciones entre los sexos, muchas veces jerárquicas, se reproducen en los procesos educativos, tanto dentro como fuera de los centros de enseñanza. Allí se desarrollan aún más y se afianzan las identidades en relación con el género. Por otra parte, las relaciones entre los sexos pueden verse desde y a través de nuevos puntos de vista, y es posible sondear en profundidad y ensayar las posibilidades de cambio. De esta forma, la educación también puede aportar de manera determinante al cuestionamiento de los modelos de pensamiento y a los roles estereotipados y discriminadores, a romper con los patrones tradicionales y a superar los prejuicios. ${ }^{10}$

Sin lugar a dudas, gran parte de la importancia de la CEDAW reside en fortalecer las nociones de universalidad e indivisibilidad de los derechos humanos. Es importante destacar que en el derecho internacional de los derechos humanos cuando hablamos de universalidad hacemos referencia a que todas las personas físicas son sujetos de derechos humanos, con independencia de su sexo, su raza, religión, condición social, ideología, etcétera. ${ }^{11}$

Del mismo modo, la Convención Interamericana Para Prevenir, Sancionar Y Erradicar La Violencia Contra La Mujer “Convención De Belem Do Pará”12

${ }^{10}$ Por ejemplo, en la recomendación general 23 "sobre la igualdad en el matrimonio y las relaciones de familia”, el comité de la Cedaw citando a la organización mundial de la salud indicó que "cuando los menores, especialmente las niñas se casan y tienen hijos, su salud puede verse afectada desfavorablemente y se entorpece su educación. Como resultado, se restringe su autonomía económica".

${ }^{11}$ La Declaración de Viena del 25 de junio de 1993 adoptada por la conferencia mundial de los derechos humanos "no admite dudas, reafirmando la universalidad, indivisibilidad e interdependencia de tales derechos", determinando que "los estados tienen el deber, sean cuales sean sus sistemas políticos, económicos y culturales, de promover y proteger todos los derechos humanos, de las libertades fundamentales".

${ }^{12}$ Hecha en la ciudad de Belem do pará, Brasil, el 9 de junio de 1994. ratificada por argentina mediante la ley 24.632 (promulgada el 1 de abril de 1996) 
y poner que ocupa rango supralegal define la violencia contra la mujer como un reflejo de la histórica desigualdad entre los sexos, y reconoce su derecho a una vida libre de violencia y declara que la violencia contra la mujer es una violación a los derechos humanos.

Este instrumento sirvió como base para que la Corte Interamericana de Derechos Humanos, en el año 2006, dictara la primera sentencia con cierta perspectiva de género en la que se reconoció la afectación específica de derechos humanos de las mujeres dentro de patrones generalizados de violencia, conocido como el caso del Penal Miguel Castro Castro vs. Perú. ${ }^{13}$

Esta convención regional es el único instrumento internacional abocado específicamente a la violencia contra la mujer y que establece además responsabilidades para los estados en caso de incumplimiento de sus postulados. ${ }^{14}$

En el caso "González y otras contra México", ${ }^{15}$ conocido como "Campo Algodonero", ${ }^{16}$ la Corte avanzó aun más al analizar el caso que involucraba la desaparición y el homicidio de tres mujeres en un contexto de violencia más amplio en el cual existían otros muchos casos que involucraban homicidios de mujeres jóvenes y de condición económica muy vulnerable.

Este caso es muy importante ya que se atribuye responsabilidad al Estado mexicano por hechos cometidos por particulares y esta responsabilidad se atribuye por la falta de la debida diligencia del estado en el cumplimiento de las pautas establecidas en la Convención Americana de Derechos Humanos y en la Convención de Belem Do Pará.

Además, la Corte avanza sobre una idea de igualdad sustancial, en la que se identifica a las víctimas en un grupo subordinado al entender de la corte, este

${ }^{13}$ Disponible en: http://www.corteidh.or.cr/docs/casos/articulos/seriec_160_esp.pdf

${ }^{14}$ En el artículo 12 del instrumento se prevé su aplicación en peticiones individuales: “cualquier persona o grupo de personas, o entidad no gubernamental legalmente reconocida en uno o más estados miembros de la organización, puede presentar a la comisión interamericana de derechos humanos peticiones que contengan denuncias o quejas de violación del artículo 7 de la presente convención por un estado parte, y la comisión las considerará de acuerdo con las normas y los requisitos de procedimiento para la presentación y consideración de peticiones estipulados en la convención americana sobre derechos humanos y en el estatuto y el reglamento de la comisión interamericana de derechos humanos."

${ }^{15}$ Disponible en: http://www.corteidh.or.cr/docs/casos/articulos/seriec_205_esp.pdf

${ }^{16}$ Para un análisis muy interesante de este caso ver artículo de Víctor Abramovich disponible en http://www.equidad.scjn.gob.mx/img/pdf/responsabilidad_estatal_por_ violencia_de_genero.pdf 
es el grupo de las mujeres en Latinoamérica. Al respecto es interesante la interpretación de la igualdad desde la perspectiva del "no sometimiento". ${ }^{17}$ Desde esta perspectiva, el Estado no solo es responsable de evitar tratos desiguales irrazonables, sino que también debe evitar la perpetuación y consolidación de situaciones en la que personas que integran determinados grupos han sido sistemática e históricamente excluidos o segregados como consecuencia de su pertenencia a esos grupos. En este sentido, el respeto por parte del Estado del valor "igualdad” no solo requiere de la omisión de acción (discriminación) del ente estatal por medio de diferencias basadas en criterios absurdos, sino que también requiere de acciones y políticas claras que tiendan a desmantelar situaciones de exclusión y segregación, algo que puede lograr, por ejemplo, a través de acciones afirmativas. ${ }^{18}$

Es importante destacar dos avances muy importantes de la Corte Interamericana en cuanto a la superación de los estereotipos de género, en los casos que comentamos anteriormente los derechos afectados eran el derecho a la vida y a la integridad personal, pero en el año 2012 en el caso Atala c/ Riffo $^{19}$ la Corte dicta una sentencia en otro contexto, un contexto no de violación sistemática de los derechos como sus precedentes sino frente a una situación de discriminación.

Esta sentencia es susceptible de múltiples enfoques, porque más allá de la incorporación del tratamiento de situaciones de discriminación de los derechos de las personas de sexualidades diversas, la corte avanza sobre desmitificar muchos estereotipos ligados a las mujeres y a la familia.

Cuando la Corte Interamericana analiza el accionar del estado chileno, concretamente la sentencia de la Corte Suprema de este país, en la que gran parte de la motivación radicaba en el hecho de que una madre debía anteponer siempre los intereses de sus hijos a los propios. ${ }^{20}$ Una vez más encontramos como, para la justicia en muchos países de la región, toma decisiones utilizando la imagen de mujer abnegada siempre ligada a la crianza de los hijos y el soste-

${ }^{17} \mathrm{Al}$ respecto, es interesante el artículo de Roberto Saba (2007)

${ }^{18}$ Para ampliar se recomienda Kemelmajer de Carlucci (2010).

${ }^{19}$ Disponible en: http://corteidh.or.cr/docs/casos/articulos/seriec_239_esp.pdf

${ }^{20}$ Recordemos que la corte suprema de chile entendió razonable denegar la custodia de sus hijas a Karen Atala porque esta reconoció públicamente su homosexualidad y decidió convivir con su pareja. 
nimiento afectivo del hogar. Cuando se presentan casos como este, cuando esa imagen proyectada no se condice con la realidad, la mirada de la sociedad en general, y por supuesto de los tribunales, suele resultar condenatoria.

En este sentido es muy interesante esta nueva mirada que nos propone la Corte Interamericana, poniendo en jaque la mira tradicional sobre los roles y opciones femeninos. Los derechos consagrados en los instrumentos internacionales de derechos humanos en general y la convención americana de derechos humanos en particular, son para todas las personas independientemente de su sexo y de su condición de madres.

Además, el fallo es muy claro en cuanto a la protección de la Convención Americana respecto del derecho de las personas a tener una familia, y ese derecho abarca no solo los modelos tradicionales de familia sino otros como el que se plantea en este caso. ${ }^{21}$

El otro caso interesante es el caso "Forneron c/ Argentina", ${ }^{22}$ que si bien es un caso en el que se ventila un caso de adopción irregular llevado a cabo en nuestro país, en el que un padre reclama la restitución de su hija biológica, la sentencia de la Corte Interamericana vuelve a mirar los estereotipos relacionados con el cuidado de los hijos, que para la justicia argentina están ligados indefectiblemente a la mujer. Para el tribunal interamericano, esas afirmaciones responden " $a$ ideas preconcebidas sobre el rol de un hombre y una mujer en cuanto a determinadas funciones o procesos reproductivos, en relación con una futura maternidad y paternidad”.

Este camino iniciado por la Corte Interamericana es muy fructífero porque sirve para afianzar la perspectiva de género en los países integrantes de la OEA, permitiendo generar la conciencia de que la afectación del derecho a la igualdad

${ }^{21}$ Considerado 142. "La corte constata que en la convención americana no se encuentra determinado un concepto cerrado de familia, ni mucho menos se protege solo un modelo "tradicional" de la misma. Al respecto, el tribunal reitera que el concepto de vida familiar no está reducido únicamente al matrimonio y debe abarcar otros lazos familiares de hecho donde las partes tienen vida en común por fuera del matrimonio". Considerando 145. "En el presente caso, este tribunal constata que el lenguaje utilizado por la corte suprema de chile relacionado con la supuesta necesidad de las niñas de crecer en una "familia estructurada normalmente y apreciada en su medio social", y no en una "familia excepcional”, refleja una percepción limitada y estereotipada del concepto de familia que no tiene base en la convención al no existir un modelo específico de familia (la "familia tradicional")".

${ }^{22}$ Disponible en: http://www.corteidh.or.cr/docs/casos/articulos/seriec_242_esp.pdf 
de las mujeres, es un problema de derechos humanos y como tal debe ser erradicado con el compromiso del Estado y por supuesto de la sociedad en general.

\section{Bibliografía}

Caputti, María (2011). “La Convención sobre la eliminación de todas las formas de discriminación contra la mujer”. En Carnota, Walter y Maraniello, Patricio (dirs.) y Leontina Sosa, Guillermina (coord). Tratado de los tratados internacionales. Comentados. t. III. Buenos Aires: La Ley.

Facio, Alda (comp.) (2000). Género y derecho. Santiago de Chile: Lom.

Facio, Alda (2007). “La carta magna de las mujeres”. Disponible en http:// www. redtrasex.org.ar/documentos/docsnuevos/facio_carta_magna_ notas_amg.pf

Kemelmajer de Carlucci, Aída (2010). “Artículo 77, inciso 23. Medidas de acción positiva”. En Sabsay, Daniel (dir.) y Manili, Pablo (coord.). Constitución de la Nación Argentina y normas complementarias. Análisis doctrinal y jurisprudencial. t. III. Buenos Aires: Hammurabi.

Lamas, Marta (2002). Cuerpo: diferencia sexual y género. México: Taurus.

Saba, Roberto (2007). «(Des) igualdad estructural». En Gargarella, Roberto y Alegre, Marcelo. El derecho a la igualdad. Aportes para un constitucionalismo igualitario. Buenos Aires: Lexis Nexis.

Ruiz, Alicia (2009). "Cuestiones acerca de mujeres y derecho”. En: Revista Aportes para el Estado y la administración gubernamental. Edición $\mathrm{N}^{\circ} 25$, Equidad de Género. Buenos Aires: Asociación de Administradores Gubernamentales. 